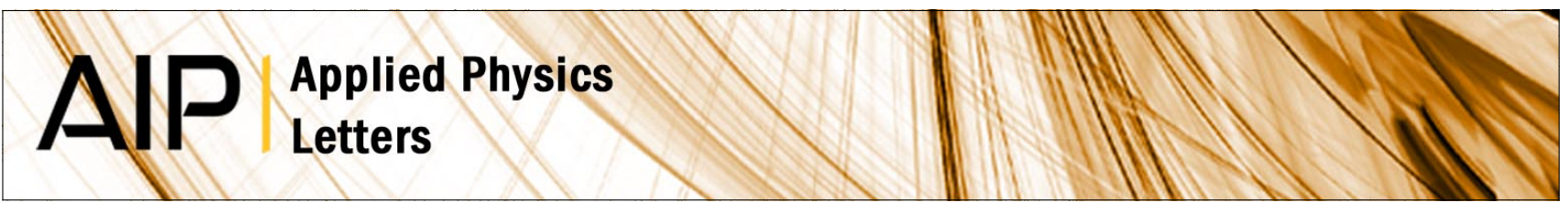

\title{
On the thermoelectric amplification of sound in semiconductors
}

M. A. Tenan, A. Marotta, and L. C. M. Miranda

Citation: Appl. Phys. Lett. 35, 321 (1979); doi: 10.1063/1.91107

View online: http://dx.doi.org/10.1063/1.91107

View Table of Contents: http://apl.aip.org/resource/1/APPLAB/v35/i4

Published by the American Institute of Physics.

Additional information on Appl. Phys. Lett.

Journal Homepage: http://apl.aip.org/

Journal Information: http://apl.aip.org/about/about_the_journal

Top downloads: http://apl.aip.org/features/most_downloaded

Information for Authors: http://apl.aip.org/authors

\section{ADVERTISEMENT}

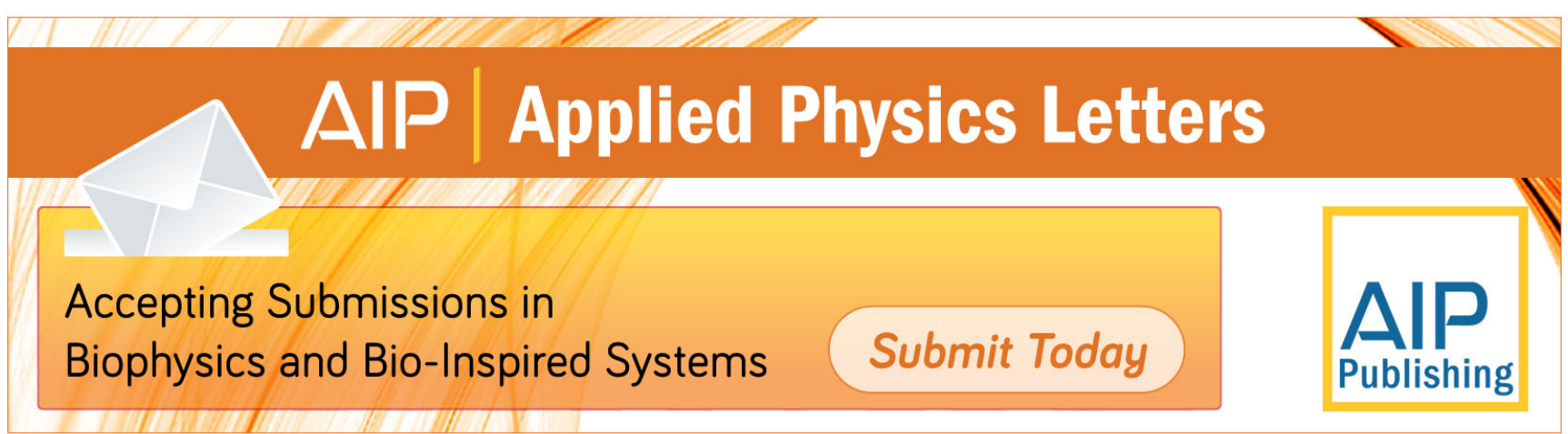




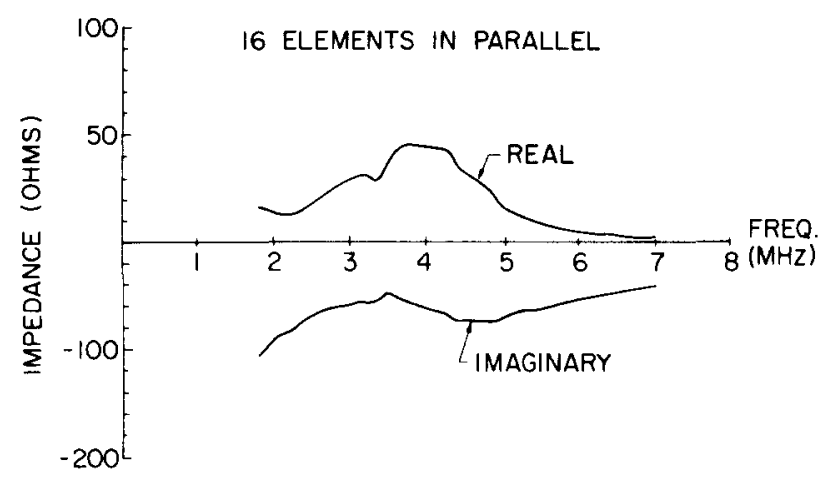

FIG. 3. The measured input impedance of 16 elements placed in parallel.

transform of this response. The elements have a center frequency of $3.1 \mathrm{MHz}$ and a $6-\mathrm{dB}$ bandwidth of $65 \%$. The insertion loss of each element was determined by exciting the element with an rf tone burst and measuring the amplitude of the reflected wave from the far end of the aluminum block. After correcting for both the reflection coefficient of a $90^{\circ}$ corner in aluminum' and the diffraction loss, the measured two-way conversion loss of each element is $14 \mathrm{~dB}$.

The input impedance of 16 elements of the array, placed in parallel, was measured and is shown as a function of frequency in Fig. 3. The insertion loss of these elements corresponds within $2 \mathrm{~dB}$ to the electrical mismatch loss when measured into a $50 \Omega$. The acceptance angle of the central element was measured by exciting it with a narrow pulse and receiving the signal with a wedge transducer that is moved along a 6-cm circle, facing the central element. The maximum amplitude of the pulse received by the wedge transducer versus angle is plotted in Fig. 4. The 6-dB acceptance angle is measured to be $\pm 35^{\circ}$. One might expect that a still broader bandwidth and wider acceptance angle could be obtained if the array were bonded to a material with a better acoustic impedance match to PZT 5H.

The surface wave generated in the substrate has been coupled onto a substrate under test by holding the two pieces parallel to each other and using a thin film of water approximately $5 \mathrm{~mm}$ long as the coupling medium. ${ }^{8}$ For aluminum-

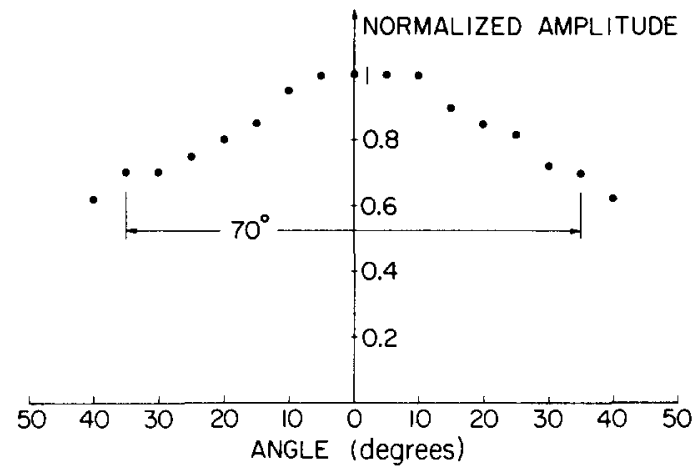

FIG. 4. The angular response of a single transducer element.

aluminum coupling, it has been calculated that at a center frequency of $3.1 \mathrm{MHz}$, the transfer loss is $2.7 \mathrm{~dB}$ with an optimum coupling length of $4.5 \mathrm{~mm}$. Experimentally, the one-way transfer loss is measured to be $3 \mathrm{~dB}$.

A new type of edge-bonded surface-acoustic-wave transducer has been built. The array has the advantages of simpler construction and use than the conventional slotted array, and furthermore should not give rise to aberrations in a focused surface wave image. High efficiency, broad bandwidth, and wide acceptance angles for the individual elements have been obtained.

This work was supported by the Advanced Research Projects Agency under Rockwell International Subcontract RI74-20773 and by the National Science Foundation grant ENG77-28528.

'C.S. DeSilets, A.R. Selfridge, and G.S. Kino, Proc. IEEE Ultrasonics Symp., 1978 (IEEE, New York), p. 111.

${ }^{2}$ B.A. Auld, M.E. Drake, and C.G. Roberts, Appl. Phys. Lett. 25, 478 (1974).

'B.A. Auld, C.S. DeSilets, and G.S. Kino, Proc. IEEE Ultrasonics Symp., 1974 (IEEE, New York, 1974), p. 24.

${ }^{4} \mathrm{C}$. Lardat and P. Defranould, Proc. IEEE 64, 627 (1976).

J.E. Bowers, B.T. Khuri-Yakub, G.S. Kino, and K-H. Yu, Ref. 1. 'E.P. Papadakis, J. Adhes. 3, 181-194 (1971).

'F. Cuozzo, E.L. Cambiaggio, J.P. Damiano, and E. Rivier, IEEE Trans. Sonics Ultrason. SU-24, 280 (1977).

${ }^{8}$ B.T. Khuri-Yakub and G.S. Kino, Appl. Phys. Lett. 32, 513 (1978).

\title{
On the thermoelectric amplification of sound in semiconductors
}

\author{
M. A. Tenan, A. Marotta, and L. C. M. Miranda \\ Instituto de Física "Gleb Wataghin", Universidade Estadual de Campinas, 13.000 Campinas-SP, Brasil
}

(Received 11 September 1978; accepted for publication 4 June 1979)

The possibility of sound amplification in a semiconductor subjected to an external temperature gradient is discussed. It is shown that for $k l<1$ and short-circuited samples, the temperature gradient threshold for amplification varies inversely proportional to $(k l)^{2}$.

PACS numbers: 72.20.Pa, 72.10.Di

Several mechanisms are known to give rise to acoustic phonon instability in semiconductors due to the electronphonon interaction. These mechanisms include, among oth- ers, the influence of a dc electric field, $, 1,2$ the direct conversion of radio waves and microwaves into acoustic waves, ${ }^{3,4}$ and the influence of a strong electromagnetic wave. ${ }^{5-7}$ More re- 
cently, however, the possibility of amplification of sound in a semiconductor subjected to a temperature gradient has been investigated by Sharma and Singh $^{8}$ using a hydrodynamic approach $(k l \ll 1 ; k$ is the sound wave number and $l$ is the electron mean free path). The semiconductor is assumed to be electrically short circuited in the direction of propagation of sound and of the temperature gradient so that the latter maintains an electric current through the sample. It is shown in Ref. 8 that the absorption of sound changes to amplification when the drift velocity of the carriers exceeds the sound velocity, i.e.,

$$
v_{d}=\tau k_{B}|\nabla T| / m \geqslant v_{s}
$$

where $v_{d}$ is the electron drift velocity due to the temperature gradient $\nabla T, k_{B}$ is the Boltzmann constant, $\tau$ is the electron relaxation time, $m$ is its effective mass, and $v_{s}$ is the sound velocity. The investigation of this "thermoelectric" amplification of sound in the opposite limiting case, $k l \gg 1$, has been done by Epshtein ${ }^{9}$ who showed that the amplification is also possible in an electrically open-circuited sample (i.e., in the absence of an electric current). The temperature gradient threshold for this amplification was found, ${ }^{9}$ for $k l \gg 1$, to depend on both the scattering mechansim and the sound frequency, and is of the same order of magnitude as the one for short-circuited conditions as given by Eq. (1). Physically, the open-circuit amplification is possible because, if $k l \gg 1$, the interaction of an acoustic wave with electrons can be regarded as the absorption and emission of phonons by individual electrons, and the probabilities of these processes depend on the electron energy. The application of a temperature gradient to a semiconductor produces a finite heat flux so that electrons drifting in one direction have a higher energy than those drifting in the opposite direction. If the external circuit is open, these "hot" and "cold" electron currents compensate each other and may eventually lead to sound amplification due to emission and absorption of phonons at different rates.

The aim of the present work is to give a detailed analysis of the thermoelectric amplification of sound in the classical regime $(k l \ll 1)$ for short-circuited samples. In particular, it is shown in the following that the temperature gradient threshold for amplification varies inversely proportional to $(k l)^{2}$ in the low-frequency region, and that the results of Ref. 8 should be understood as a high-frequency limit of the classical approach. We assume the sample is short circuited in the direction of the temperature gradient ( $z$ direction) and that the phonon wavelength is much greater than the DeBroglie wavelength of the electrons, so that their motion may be treated classically. The Boltzmann equation for the electrons interacting with an acoustic wave of frequency $\omega$ propagating in the direction of the temperature gradient can be written as ${ }^{10}$

$$
\frac{\partial f}{\partial t}+\mathbf{v} \cdot \frac{\partial f}{\partial \mathbf{r}}-\frac{e}{m} \mathbf{E}_{s} \cdot \frac{\partial f}{\partial \mathbf{v}}=-\frac{f-f^{(0)}\left(1+n_{s} / n_{0}\right)}{\tau}
$$

where $\mathbf{E}_{s}$ is the longitudinal self-consistent electric field induced by the acoustic wave, $f^{(0)}$ is the local equilibrium Maxwellian distribution function, and $n_{0}$ and $n_{s}$ are the equilib- rium value and the fluctuating part $\{\sim \exp [i(\mathbf{k} \cdot \mathbf{r}-\omega t)]\}$ of the electron density, respectively. The relaxation time $\tau$ is assumed to be energy independent. The linearized solution of Eq. (2) is obtained by writing the electron distribution function in the form

$$
f=f^{(0)}+\tilde{f}+g \exp [i(\mathbf{k} \cdot \mathbf{r}-\omega t)] .
$$

In Eq. (3), the second term is the static component of the small deviation from the local equilibrium due to the temperature gradient, whereas the third term represents the oscillating component induced by the acoustic wave. The static distribution can be found, to first order in $\nabla T$, by solving the static form of Eq. (2), and the result is as follows:

$$
\tilde{f}=\left(\frac{v^{2}}{v_{T}^{2}}-\frac{3}{2}\right) \frac{2 \mathbf{v} \cdot \mathbf{v}_{d}}{v_{T}^{2}} f^{(0)},
$$

where

$$
v_{T}=\left(2 k_{B} T / m\right)^{1 / 2}
$$

and

$$
\mathbf{v}_{d}=-\frac{k_{B} \tau}{m} \frac{\partial T}{\partial z} \hat{z}
$$

The other equations which describe the electron gas in the presence of an acoustic wave are the modified Poisson's equation and the equation of motion of the lattice, namely,

$$
\frac{\partial E_{s}}{\partial z}=-\frac{4 \pi e n_{s}}{\epsilon}+\frac{4 \pi \beta}{\epsilon} \frac{\partial^{2} u}{\partial z^{2}}
$$

and

$$
\rho \frac{\partial^{2} u}{\partial t^{2}}=C \frac{\partial^{2} u}{\partial z^{2}}-\beta \frac{\partial E_{s}}{\partial z}
$$

where $\epsilon$ is the static dielectric constant, $\beta$ is the coupling constant, $\rho$ is the crystal density, and $C$ is the appropriate elastic constant.

Fourier transforming Eqs. (6) and (7) and using Eqs. (2)-(4) to eliminate $n_{s}$ one obtains the following dispersion relation:

$$
\frac{v_{d}}{v_{T}}=\frac{2 Y_{1}-i k l\left(1-Y_{4}\right) Q}{4\left(Y_{2}-Y_{3}\right)},
$$

where

$$
\begin{aligned}
& Q=\frac{1}{\omega_{p}^{2} \tau^{2}} \frac{\omega^{2}-\left(v_{s}^{2}-\alpha^{2}\right) k^{2}}{\omega^{2}-v_{s}^{2} k^{2}}, \\
& \alpha^{2}=4 \pi \beta^{2} / \rho \epsilon, \\
& \omega_{p}^{2}=4 \pi e^{2} n_{0} / \epsilon m, \\
& Y_{1}=\frac{1}{n_{0}} \int d^{3} v \frac{v_{z}}{v_{T}} \frac{f^{(0)}}{1+\Omega}, \\
& Y_{2}=\frac{1}{n_{0}} \int d^{3} v\left(\frac{v^{2}}{v_{T}^{2}}-\frac{3}{2}\right) \frac{f^{(0)}}{1+\Omega}, \\
& Y_{3}=\frac{1}{n_{0}} \int d^{3} v \frac{v_{z}^{2}}{v_{T}^{2}}\left(\frac{v^{2}}{v_{T}^{2}}-\frac{5}{2}\right) \frac{f^{(0)}}{1+\Omega}, \\
& Y_{4}=\frac{1}{n_{0}} \int d^{3} v \frac{f^{(0)}}{1+\Omega},
\end{aligned}
$$




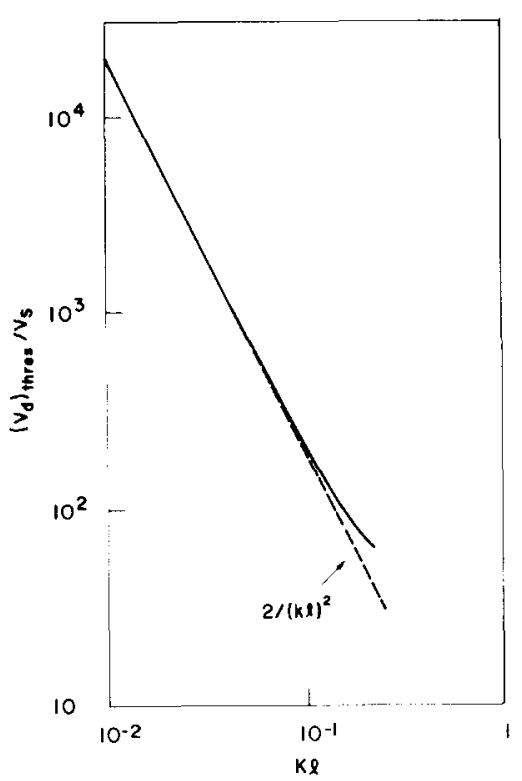

FIG. 1. Variation of the threshold value for the drift velocity as a function of $k l$ for a sample of InSb at room temperature. The full line is the result of the numerical calculation whereas the dashed line corresponds to the expres$\operatorname{sion}\left(v_{d} / v_{s}\right)_{\text {thresh }}=2 /(\mathrm{kl})^{2}$. The sound wave was assumed to be propagating along the [111] direction $\left(v_{\mathrm{s}}=3.89 \times 10^{5} \mathrm{~cm} / \mathrm{s}\right)$.

with

$$
\Omega=i\left(k v_{z}-\omega\right) \tau \text {. }
$$

In Eqs. (8)-(11) $\omega$ is, in general, complex, i.e., $\omega=\omega_{R}+i \omega_{I}$. When $\omega_{I}>0$, the wave is amplified. The condition for switching over from attenuation to amplification is obtained by putting $\omega_{I}=0$, i.e., $\omega=\omega_{R}$. This condition gives the threshold value of $\partial T / \partial z$ required for the amplification of the wave. The explicit expression for the dispersion relation is, in general, quite complicated due to the dependence of Eq. (8) on the $Y$ integrals, Eq. (10). The $Y$ integrals, however, can all be expressed in terms of the error function " and therefore readily calculable by numerical methods. Nevertheless, in the limiting case of $k l \ll 1$ and $\omega \tau \ll 1$, a simple analytical form for both the dispersion relation and the threshold value of $|\nabla T|$ can be obtained by using the asymptotic representation for the error function $\Phi(z),{ }^{11}$ namely,

$$
\Phi(z)=1-\frac{e^{-z^{\prime}}}{\pi}\left(\sum_{m=0}^{n} \frac{(-1)^{m} \Gamma\left(m+\frac{1}{2}\right)}{z^{2 m+1}}+R_{n}\right)
$$

Performing the calculations and putting $\operatorname{Im} \omega=0$ in Eqs. (8)-(10), the result one obtains in this case is then

$$
|\nabla T|_{\mathrm{thr}}=\frac{m v_{s}}{k_{B} \tau} \frac{2}{(k l)^{2}},
$$

so that

$$
\frac{v_{d}}{v_{s}}=\frac{\tau k_{B}|\nabla T|}{m v_{s}} \geqslant \frac{2}{(k l)^{2}} \geqslant 1 .
$$

In Fig. 1 we plot the threshold value for $\left(v_{d}\right)_{\text {thresh }}$ as a function of $k l$, obtained from numerical calculations and from Eq. (12) for a typical sample of InSb. Figure 1 together with
Eqs. (12) and (13) first suggest that the results of Ref. 8 are the high-frequency limit of the classical approach. Second, the thermoelectric amplification, in the low-frequency region, is frequency dependent, i.e., for a fixed value of $\mid \nabla T$ only certain modes with frequencies in a given interval [Eq. (13)] will become unstable. For very small values of $k l$, the temperature gradient needed becomes forbiddingly large. To our knowledge this is the first frequency-selective sound amplification mechanism reported so far. All the previous mechanisms mentioned in the introduction ${ }^{1-7}$ have a frequency-independent threshold. Physically, this frequencydependent threshold for the thermoelectric amplification of sound may be interpreted as a result of the energy exchange between the carriers and the mechanical vibrations as follows. The power acquired by the carriers at the threshold is about

$$
m v_{d}^{2} / 2 \tau
$$

This electrical power comes from the interaction of the carriers with the mechanical vibrations induced by the temperature difference established in the sample. The excess of thermal (mechanical vibration) power of an acoustic phonon of frequency $f$ available in one electron mean free path is about $k_{B} \Delta T f$. The effective power transferred to a single electron is then given by

$$
\eta k_{B} \Delta T f=\eta k_{B}|\nabla T|_{\mathrm{thr}} l f
$$

where $\eta$ is an energy conversion efficiency for one electron. This efficiency $\eta$ should, in a first approximation, be roughly proportional to the inverse of the number of electrons in one acoustic wavelength, i.e., $\eta \sim l / \lambda$. Hence, equating Eqs. (14) and (15), and using Eq. (5b) and $\eta \sim l / \lambda$, one obtains

$$
|\nabla T|_{\mathrm{thr}}=\frac{m v_{s}}{2 k_{B} \tau}\left(\frac{\lambda}{l}\right)^{2} \sim \frac{m v_{s}}{k_{B} \tau} \frac{2}{(k l)^{2}},
$$

which agrees with the result of Eq. (12); i.e., in the short time scale where the heat transport is carried by the acoustic phonons rather than by diffusion, the power acquired by the electrons is essentially dependent on the relative length of the carrier mean free path to the acoustic phonon wavelength $(l / \lambda)$. For a very long wavelength compared to the electron mean free path, the power acquired by one electron is very small. Hence, to obtain a high drift velocity the gradient has to be extremely large, as shown in Eq. (12).

'A.R. Hutson, J.H. McFree, and D.L. White, Phys. Rev. Lett. 7, 237 (1961).

${ }^{2}$ H.N. Spector, Solid State Phys. 19, 291 (1966).

${ }^{3}$ R. Abeles, Phys. Rev. Lett. 19, 1181 (1967); A. Zemel and Y. Goldstein, Phys. Rev. B 7, 191 (1973).

${ }^{4}$ L.C.M. Miranda, Phys. Lett. A 46, 25 (1973).

'L.C.M. Miranda, J. Phys. C 9, 2971 (1976).

${ }^{6}$ L.C.M. Miranda, Solid State Commun. 23, 219 (1977).

'E.M. Epshtein, Sov. Phys.-Solid State 11, 2213 (1970).

${ }^{8}$ S. Sharma and S.P. Singh, J. Appl. Phys. 45, 4656 (1974).

${ }^{9}$ E.M. Epshtein, Sov. Phys.-Semicond. 9, 1043 (1976)

${ }^{10}$ H.N. Spector, Phys. Rev. 165, 562 (1968).

${ }^{1}$ I.S. Gradshteyn and I.M. Ryzhik, Table of Integrals, Series and Products (Academic, New York, 1965), p. 931. 